\title{
MultiSpectral Image Binarization using GMMs
}

\author{
Fabian Hollaus, Markus Diem and Robert Sablatnig \\ Computer Vision Lab \\ TU Wien \\ Vienna, Austria \\ holl@cvl.tuwien.ac.at
}

\begin{abstract}
MultiSpectral Imaging enhances the study of degraded historical documents. It allows for visualizing washed out or even invisible ink but also improves the automated analysis because of a denser spectral sampling. We present a new methodology for binarization of multispectral document images that groups spectral signatures of different sources by fitting two Gaussian Mixture Models (GMMs) with Expectation Maximization. Both GMMs assign cluster labels to the multispectral samples and the clustering results are combined for the identification of the handwriting regions. The method is evaluated on the ICDAR 2015 MS-TEx dataset. Results on this publicly available benchmarking set are encouraging.

Index Terms-MSI, binarization, GMM
\end{abstract}

\section{INTRODUCTION}

Multi- and hyperspectral imaging have been recently applied successfully in the field of document image analysis. MultiSpectral Imaging (MSI) is a non-invasive analysis method that is capable of detecting features that are invisible to the human eye: Thus, it can be used to increase the legibility of faded-out or erased texts in ancient manuscripts [1]. Other applications of this non-invasive investigation method are the differentiation between ink types in hyperspectral images [2] or the binarization of ancient document images [3].

Document image binarization is typically used as preprocessing step for document analysis systems such as optical character recognition [4]. Historical documents can be corrupted by bleeding artifacts, background clutter or variation or can contain faded-out ink [4]. These degradations impede the binarization task, compared to the binarization of modern handwriting images and several methods have been proposed to overcome these challenges. For instance in [5] a Fully Convolutional Network (FCN) operating at different image scales is used for foreground segmentation. The inputs for the FCN are unprocessed grayscale document images and the output of the binarization method in [6]. The proposed method achieved the second rank at the 'ICDAR 2017 Competition on Document Image Binarization' contest [7]. Howe [6] suggests a Markov Random Field (MRF) model in which a graph cut is applied on the Laplacian of the image intensity. Another binarization method is suggested by Su et al. in [4]. The

Personal use of this material is permitted. Permission from IEEE must be obtained for all other uses, in any current or future media, including reprinting/republishing this material for advertising or promotional purposes, creating new collective works, for resale or redistribution to servers or lists, or reuse of any copyrighted component of this work in other works.

978-1-5386-5875-8/18/\$31.00 @2018 IEEE. method makes use of local image contrasts and the width of the handwriting strokes. In [8] a binarization framework is proposed that makes use of Local Co-occurrence Mapping, local contrasts and GMM. The GMM used is applied on grayscale images, whereas our aim is to binarize multispectral images.

Several methods have been proposed for the binarization of multispectral document images: In [9] a higher order MRF is used for the classification of degraded handwritings imaged with a MSI system. Another binarization method [10] combines Independent Component Analysis [11] with an image fusion technique. In [12] a multiple-expert binarization framework is suggested that combines subspace selection with state-of-the-art binarization methods for grayscale images (including the technique in [6]). The multiple-expert binarization framework is applied on a dataset that is used in the 'ICDAR 2015 MultiSpectral Text Extraction (MS-TEx 2015)' [3] contest. The five participating methods of this contest are especially designed for multispectral document images and achieved a better performance than a conventional binarization method [6] that is applied on single channels of the multispectral scan. The winning method of the competition combines a matched filter technique (namely the Adaptive Coherence Estimator [13]), with the grayscale binarization method proposed in [4] and the GrabCut algorithm [14].

The binarization framework for multispectral document images proposed in this work makes use of GMM's for the clustering of the MSI datasets introduced in [3]. The GMM's are used to separate the handwriting from other classes: These classes are stamps, annotations, degradations and surrounding background regions [3]. For this purpose, two GMM based clustering steps are used: The first clustering step is used to identify the classes contained in the MSI data and the second clustering step is used to refine the clustering of the handwriting. Finally, both clustering steps are combined for the handwriting extraction. While only the handwriting extraction is evaluated numerically, resulting images provided in this work show that the GMM's are also capable of identifying the other classes. To the best of our knowledge, this is the first time that GMMs have been applied on multi- or hyperspectral images of documents.

This work is structured as follows. In Section II the binarization framework is introduced. The method is then evaluated in Section III. Finally, a conclusion is given in Section IV. 


\section{Methodology}

\section{A. Gaussian Mixture Models}

We use GMM's for the clustering of the multispectral document images. Thereby, each sample of the $d$ - dimensional multispectral image data is modeled by a probability density function $h(\mathbf{x})$ at the locations $\mathbf{x} \in \mathbb{R}^{d}$ by the following equation:

$$
h(\mathbf{x})=\sum_{i=1}^{N} \alpha_{i} \mathcal{N}\left(\mathbf{x} \mid \mu_{i}, \boldsymbol{\Sigma}_{i}\right),
$$

whereby $\mathcal{N}$ denotes a normal density function with the covariance $\boldsymbol{\Sigma}_{i}$ and the mean $\mu_{i}$. A weighting factor $\alpha_{i}$ is assigned to each of the $N$ Gaussians, whereby $\sum_{i=1}^{N} \alpha_{i}=1$.

The parameters that have to be learned are $\boldsymbol{\Sigma}_{i}, \mu_{i}$ and $\alpha_{i}$ for each of the $N$ components. The parameter set is learned with the Expectation Maximization (EM) algorithm, which increases the likelihood of the learned model in an iterative manner. In our application the EM algorithm is applied once and not further repeated. The maximum number of iterations is set to 500, because the EM algorithm converges within this number of iterations in each of the conducted experiments. The number of components in our setup is set to $N=10$.

In the proposed work, GMM's are used for clustering. For this clustering purposes, for each observation $\mathbf{x}$ the posterior probability that the data point is described by a certain GMM component is calculated. Afterwards the data point is assigned to the mixture component for which the highest posterior probability is achieved.

It should be noted that the output of the GMM depends on the initialization of the parameter sets. In the GMM implementation used, the parameters are initialized based on the clustering results of the k-means++ algorithm [15]. Figure 1 (middle) shows a clustering output of this k-means extension. It is notable that the stamp and the foreground classes are assigned to multiple clusters and that both classes are not correctly separated ${ }^{1}$. Figure 1 (right) shows the output of a GMM. It can be seen that the different classes (stamp, handwriting and bleed through) are better separated than in the $\mathrm{k}$-means result. Both clustering results have been obtained on images that have been preprocessed by the procedure described in the following.

\section{B. Preprocessing}

Applying the GMM framework on unprocessed MSI data is error-prone, because the GMM's model background variations by generating own components for such regions, which impedes a correct binarization. Figure 2 (left) shows an example for this circumstance, where the background is modeled by 5 components. The foreground is instead modeled by two Gaussian components (colored black and cyan). These two components are not only assigned to the foreground but

\footnotetext{
${ }^{1}$ The clustering results shown in this work are color coded, whereby the color coding was chosen manually for each result in order to provide a vivid representation of the clustering output and to achieve a sufficient contrast between the different classes.
}

also to two background regions in the left image part. The foreground background separation is additionally impeded by the overlapping background regions.

In order to overcome this drawbacks, we make use of a preprocessing step that lowers the influence of background variations on the outcome of the GMM's. The preprocessing is applied separately on each channel $I_{i}$ of the $d$-dimensional multispectral image set. In a first step the input image $I_{i}$ is filtered with a two dimensional median filter. The resulting image $I_{m_{e} d_{i}}$ exhibits solely large background regions. The size of the median filter is chosen in such a manner that foreground pixels are filtered out by the median filter and the resulting image is solely dependent on the intensity of the background pixels. In our case a filter size of $73 \times 73 p x$ is used. This filter size was selected manually based on the investigated dataset. It was found that for the datasets examined the resulting images $I_{\text {med }_{i}}$ exhibit only background regions. The overall resulting image $I_{f_{i}}$ is simply computed by subtracting the median filtered image from the input image:

$$
I_{f_{i}}=I_{i}-I_{\text {med }_{i}}
$$

The resulting images $I_{f}$ are then used as inputs for the GMM based clustering. By using this simple preprocessing step, the influence of background variations is reduced, as can be seen in the example in Figure 2 (right). The background is more uniform compared to the result gained on unprocessed images. Additionally, foreground regions that are not found in Figure 2 (left) are correctly labeled as belonging to the foreground.

\section{Clustering with k-means Initialization}

The preprocessed images are clustered with a GMM, whereby the initial parameter values used by the EM algorithm are found by the k-means++ clustering algorithm [15]. One covariance matrix is shared by the Gaussians and the covariance matrix is regularized by a value of 0.00001 . By using a shared covariance matrix the influence of background noise is lowered as can be seen in Figure 3, where the output in Figure 3 (left) is gained by a GMM with a shared covariance and the resulting image in Figure 3 (right) is obtained by a GMM with separate covariance matrices. The latter one exhibits more background clutter and bleeding artifacts. The regularization of the covariance matrix is used to further lower the influence of background noise.

In the majority of the MSI sets analyzed, the handwriting is modeled by two Gaussians, whereby one Gaussian describes darker handwriting regions and the second component describes brighter handwriting regions. The darker regions are typically located at thicker stroke regions, whereas the brighter regions are located at thinner stroke regions. This can also be seen in Figure 3, where the darker and brighter regions identified by the GMM's are colored cyan and orange (in Figure 3 (left)) and blue and red (in Figure 3 (right)). In the following, such darker and thicker handwriting regions are referred to as dominant foreground regions. The brighter 

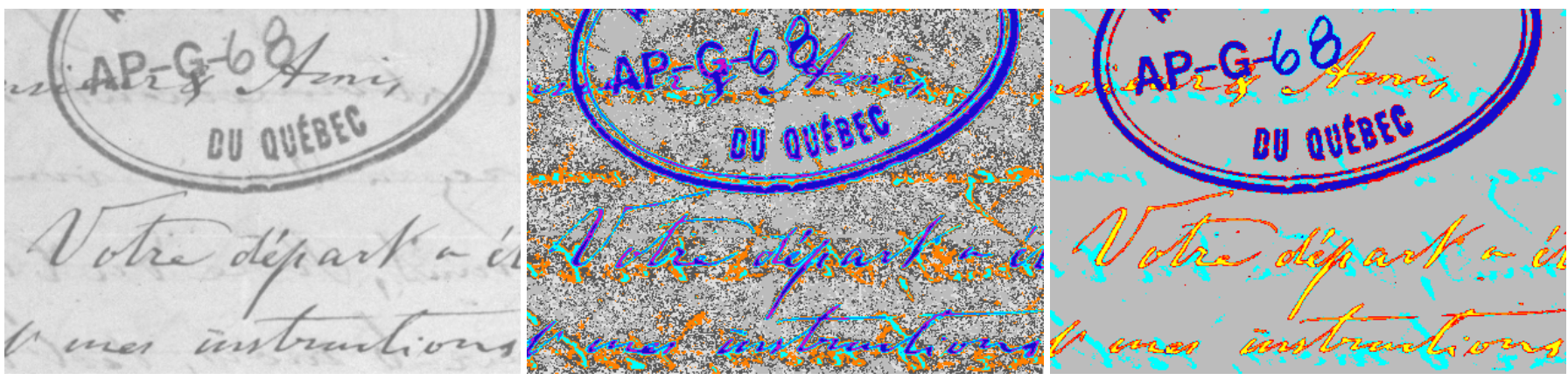

Fig. 1. Clustering using k-means and GMM. (Left) Grayscale image taken from the multispectral image data. (Middle) k-means++ clustering result using 10 components. (Right) GMM result using 10 components.
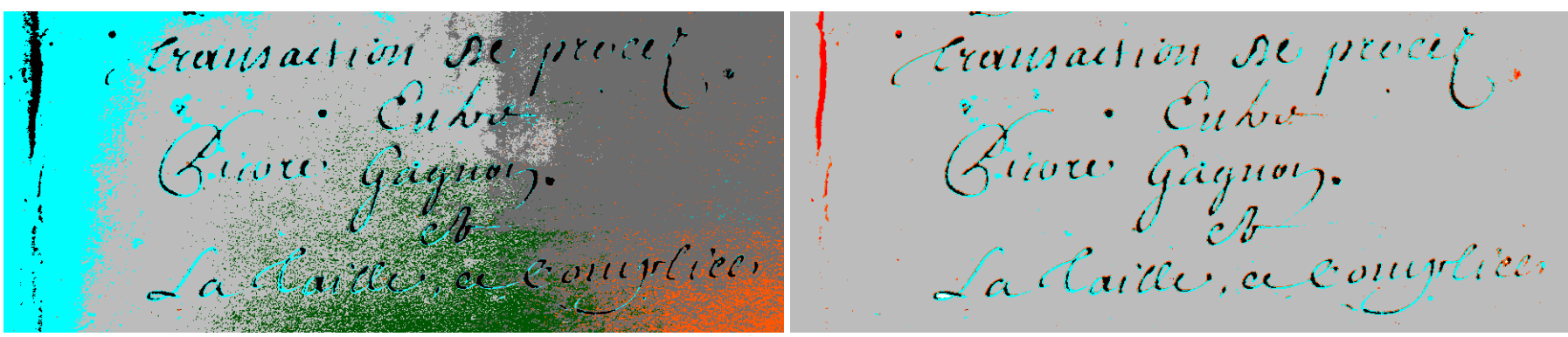

Fig. 2. Effect of the filtering of the multispectral images. (Left) GMM clustering result obtained on unprocessed data. (Right) Result obtained on images that have been filtered with a median filter. For both results the number of components is set to 10 .

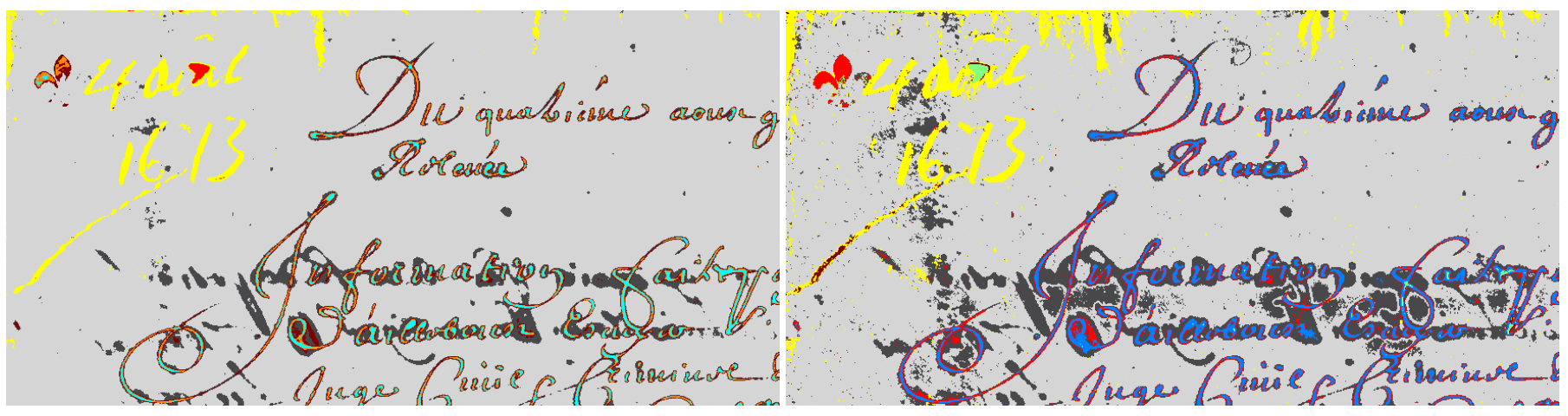

Fig. 3. Effect of the choice of the covariance. (Left) Output by a GMM with a single covariance shared by all 10 components. (Right) Result obtained by a GMM with different covariances for each of the 10 components. The writing colored yellow is an annotation and is correctly separated from the handwriting. Bleed through artifacts are colored in dark gray.

regions are so called mixed pixels - in terms of spectral unmixing - because their spectral signatures are mixtures of the spectral signatures of dominant foreground regions and the spectral signatures of surrounding background regions. It should be noted that these mixed pixels are not as well separable as so called pure pixels (belonging for instance to dominant handwriting regions). This can also be seen in Figure 3 , where the small artifact in the upper left corner is assigned to the cluster that describes mainly such mixed pixels.

This circumstance and the fact that the foreground is in the majority of the test cases modeled by two components impedes a correct binarization. Since the foreground is modeled by multiple components, we do not take the outcome of the GMM as final result, but instead apply a second clustering step and combine the outputs of both GMM's.

\section{Clustering with Estimated Initialization Values}

The parameter value initialization of the second GMM clustering step depends on the outcome of the first GMM clustering step. The different object classes (foreground, background, stamps etc.) are typically correctly separated from each other by the first GMM clustering step. Hence, the mean values $\mu_{i}$ and covariances $\boldsymbol{\Sigma}_{i}$ of the Gaussian components found are used for the initialization of the second GMM clustering step.

This parameter initialization is performed for all components found, except for two cases: (1) If the number of pixels belonging to a cluster is small than a predefined threshold, 
the component is not used in the second clustering step. The clusters that are rejected by this step are corresponding to small regions containing background variations or to sensor / image noise. In our case we use a threshold of 200px. The threshold is selected manually in order to remove the sensor / image noise that is partially contained in the investigated datasets. (2) The cluster describing bright and thin handwriting stroke regions is also rejected. Hence, the Gaussian component describing the handwriting is simply initialized with the parameters found for the dominant handwriting class. The component that models the bright handwriting regions is rejected, because of two reasons. First, the component is describing mixed pixels, which are not as well separated from other classes as pure pixels. Second, we found that these mixed-pixels can be omitted for the parameter initialization, because the EM algorithm converges to a solution in which these mixed pixels belonging to the foreground are correctly labeled as belonging to the same cluster as the dominant writing regions.

In order to identify pixels belonging to the dominant handwriting class and mixed-pixels, the following procedure is applied: First, a single channel of the multispectral images acquired at $500 \mathrm{~nm}$ is binarized with the method of $\mathrm{Su}$ et al. [4]. Afterwards, the skeleton of the binarization result is computed and multiplied with the clustering result. The remaining foreground pixels are counted and the dominant writing class is found by finding the class that possesses the most foreground pixels. By using the skeleton instead of the binarization result, the stroke lengths are analyzed instead of the stroke areas. Thus, the influence of classes occupying relative large regions (like stamps) on the identification of the dominant foreground class is lowered.

In the second step, the Gaussian component that describes the bright and thin stroke regions is identified: The connected components in the binarization output of $\mathrm{Su}$ et al. [4] that contain at least one pixel labeled as dominant writing region are considered. The class that is most often found in these connected components (except of course the dominant handwriting class) is assumed to describe mainly the bright and thin stroke regions.

An example for the two clustering steps is given in Figure 4. It can be seen that in the output of the first clustering step - shown in Figure 4 (middle) - the handwriting is modeled by three components. Contrary, in the output of the second clustering step - shown in Figure 4 (right) - the text is mainly modeled by one component. Additionally, in this image bleedthrough artifacts are modeled by an own component. This circumstance can be attributed to the fact that in the first clustering result the writing is modeled by three components. The procedure dedicated to the identification of the dominant writing class and the second writing class just makes use of two classes. The third class modeled by the GMM shown in Figure 4 (middle) is instead used for the initialization of the second GMM. The spectral signature of this class is similar to the spectral signature of the bleed-through and the EM algorithm converges to a solution in which the bleed- through is labeled as an own cluster. While this example shows the capabilities of the GMM for the identification of different classes, it also shows the importance of an adequate initialization.

\section{E. Final Foreground Segmentation}

In the final step of the binarization framework both GMM clustering results are combined. The result of the first clustering step is less corrupted by background clutter, compared to the output of the second clustering step, but the text is modeled by multiple components. Hence, the output of the first clustering stage is used to eliminate background clutter. Therefore, a mask is generated that is encoding foreground regions that are different to the background. One example for such a mask is given in the first image in Figure 5. The image exhibits handwriting regions and additionally a stamp. The images in the Figure 5 are color coded, whereby green indicates true positives, red depicts false positives and black indicates false negatives.

The output of the second clustering step is used to create a mask that is encoding the regions that are assigned to the Gaussian component that is modeling the handwriting class. The second image in Figure 5 shows such a mask that is based on the output of the second clustering step.

Both masks are then multiplied. Additionally, the output of the grayscale binarization algorithm in [4] is combined with the multiplication result of both masks: Therefore, the connected components found by the binarization algorithm in [4] are only added to the resulting image if they are connected to the multiplication result of both masks. Thus, stroke endings found by the binarization algorithm in [4] but not by both clustering steps are added to the final result. The third image in Figure 5 shows the output of the binarization algorithm [4] and the fourth image in Figure 5 shows the final result.

\section{RESULTS}

The binarization is evaluated on two datasets that have been used in the ICDAR 2015 MS-TEx competition [3]. The first dataset is hereafter named MS-TEx 1 dataset and has been provided to the participants of the competition for training purposes. It contains 21 portions of multispectral document images. The second dataset is hereafter referred to as MS-TEx 2 dataset and has been used as test set in the ICDAR 2015 MSTEx competition. The MS-TEx 2 dataset contains 10 portions of multispectral document images. The handwriting contained in both datasets is written with iron gall ink. Both datasets contain images that exhibit at least a handwriting class and a background class. Additionally, the images exhibit partially stamps, annotations and degradation classes. The ground-truth data encodes solely handwriting and background classes, since the overall aim of the database is to evaluate the text extraction.

Each multispectral stack consists of 8 grayscale images, which have been imaged in narrow-band spectral ranges, ranging from $340 \mathrm{~nm}$ until $1100 \mathrm{~nm}$. The performance is quantified with Precision (P), Recall (R), F-Measure (F) and 

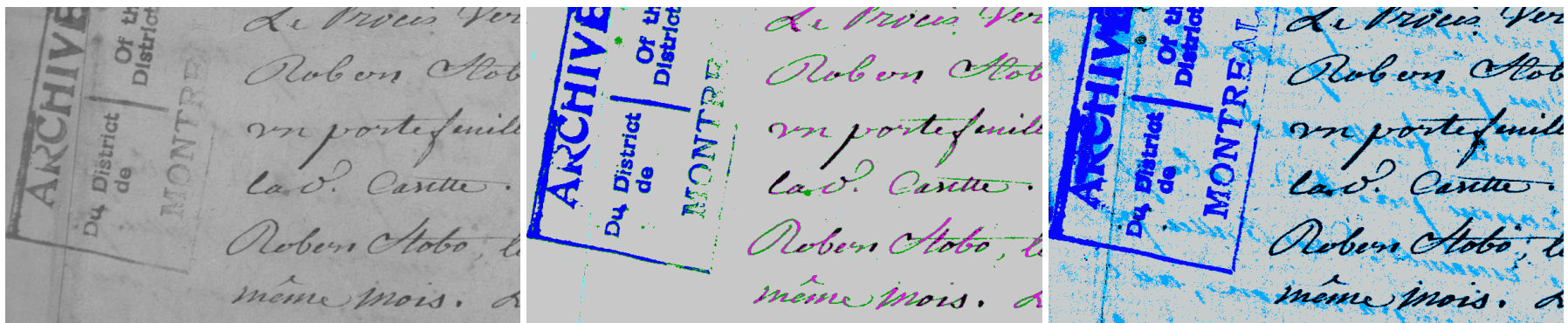

Fig. 4. GMM based clustering. (Left) Grayscale image taken from the multispectral image data. (Middle) Result of the first clustering step. (Right) GMM result of the second clustering step.
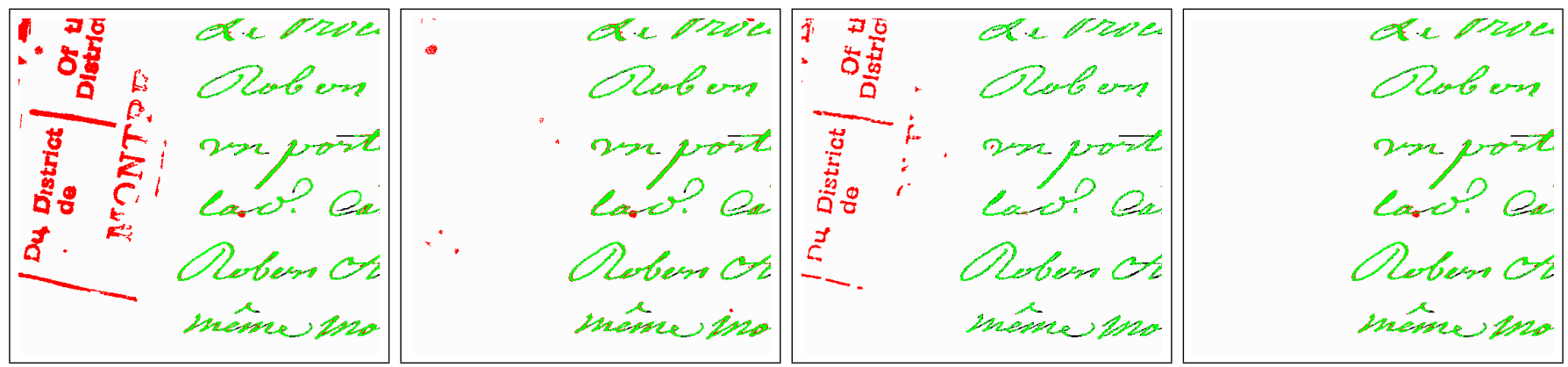

Fig. 5. Different stages of the binarization framework. From left to right: Mask generated based on the first clustering stage. Mask generated based on the second clustering stage. Output of the binarization method in [4]. Final output.

TABLE I

AVERAGE PERFORMANCE GAINED ON THE MS-TEX 1 DATASET.

\begin{tabular}{cccccc} 
Approach & F & R & P & NRM & DRD \\
\hline Proposed & 82.99 & 87.84 & 79.73 & 6.65 & 4.72 \\
\hline Diem et al. [16] & $\mathbf{8 7 . 0}$ & 86.1 & 88.1 & 7.33 & 3.09 \\
\hline Hollaus et al. [17] & 85.80 & 84.48 & 87.65 & 8.11 & 3.51 \\
\hline Moghaddam et al. [12] & 80.81 & - & - & - & - \\
\hline
\end{tabular}

Distance Reciprocal Distortion Metric (DRD) as defined in [7] and Negative Rate Metric (NRM) as defined in [12]

\section{A. MS-TEx 1}

The overall performance of the binarization framework is provided in Table I. The proposed method achieved an average F-Measure of 82.99 , which is lower than the performance gained by the methods in [16] and [17], but higher than the performance of the method in [12].

\section{B. MS-TEx 2}

The performance in terms of F-Measure gained on the MSTEx 2 dataset is given in Table II, where it is compared to the two methods that were placed first and second in the MSTEx 2015 contest. The MS-TEx 2 dataset is smaller than the MS-TEx 1 dataset, but it is more challenging, since the performance of all three approaches is smaller compared to the results gained on the MS-TEx 1 dataset. It can be seen that the method proposed achieves the second highest performance.

In Figure 6 the performance in terms of F-Measure is compared to the results gained by the participants of the MS-TEx contest [3]. The last bar depicts the performance

\begin{tabular}{cccccc} 
Approach & F & R & P & NRM & DRD \\
\hline Proposed & 82.69 & 87.44 & 79.61 & 7.11 & 4.70 \\
\hline Diem et al. [16] & $\mathbf{8 3 . 3 3}$ & - & - & 9.25 & 4.24 \\
\hline Hollaus et al. [17] & 81.90 & - & - & 10.1 & 4.74 \\
\hline
\end{tabular}

TABLE II

AVERAGE PERFORMANCE GAINED ON THE MS-TEX 2 DATASET.

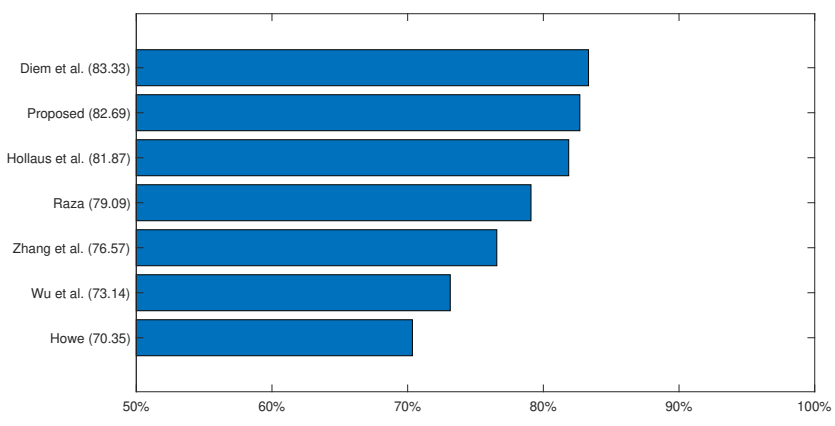

Fig. 6. Average performance gained on the MS-TEx 2 dataset. The method is compared to results gained in the MS-TEx contest [3].

gained by the binarization method introduced in [6], which is designed for grayscale images. It can be seen that all methods designed for MSI data achieve a higher performance than the approach for grayscale images.

Finally, it is evaluated how the number of Gaussian components $N$ influences the overall binarization performance. In Figure 7 the results gained with a varying number of components - from 4 to 14 - is given. The highest performance 


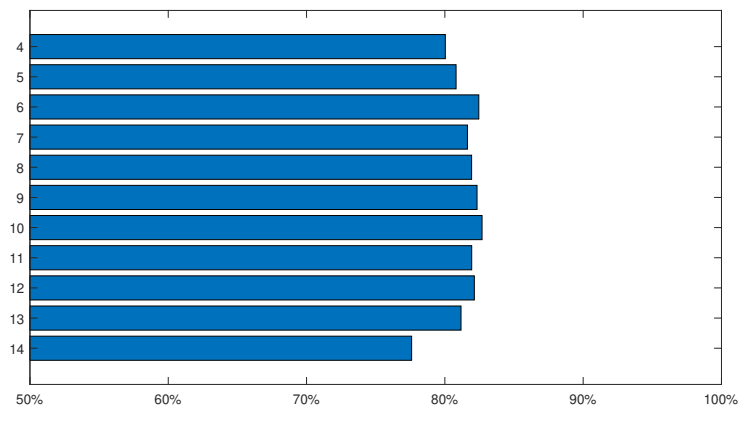

Fig. 7. Evaluation of the influence of the number of components (in terms of F-Measure). The number of components is given on the y-axes.

- namely $82.69 \%$ - is achieved when using $N=10$. It can be seen that the performance is significantly lower if 4 or 14 components are used, since this leads to decreased F-Measure values of 80.33 and 77.60. While using $N=4$ is to low for the GMM's to separate the different classes, $N=14$ results in over-fitted models. Thus, the handwriting is modeled by 3 or more components and the procedure introduced in Section II-D for the determination of the handwriting class is insufficient, because it is actually designed for two classes.

\section{CONCLUSiON}

In this work a binarization method for multispectral document images is proposed. The algorithm makes use of GMM based clustering. It is shown that applying the GMM framework on unprocessed MSI data is error prone, because the Gaussian components model background variation. We suggest a simple preprocessing step that is based on median filtering in order to remove such background variations and to improve the overall binarization performance. The processed images are used as inputs for the GMM based clustering. Since the foreground class is modeled by multiple Gaussians a second GMM based clustering is performed in order to refine the clustering result. The parameters used in the second clustering are initialized based on the output of the first GMM clustering. The outputs of both clustering steps are afterwards combined.

The method is evaluated on two datasets, where the method achieves encouraging results. However, the method is outperformed by two approaches that identify the spectral signatures of the handwriting and the background class. Contrary, the GMM based method proposed can also be used to identify other object classes. We are planning to evaluate this detection of other classes, by enriching the ground truth data with an annotation of these classes.

In the future we will make use of spatial information. It is shown in [16] that using the GrabCut algorithm [14], which makes use of spatial information, improves the binarization performance.

The binarization method is evaluated on MSI data containing eight different channels. This is a relatively small number of channels compared to other MSI systems like the one in [1] or compared to hyperspectral imaging systems for documents
[2]. We are planning to evaluate how the system performs on datasets containing a larger number of channels and if dimension reduction methods are useful for such datasets.

\section{ACKNOWLEDGEMENTS}

The authors wish to thank Rachid Hedjam and Mohamed Cheriet for providing their dataset to the public.

This research was funded by the Austrian Federal Ministry of Science, Research and Economy, by the European Union's Horizon 2020 research and innovation programme under grant agreement No 674943 (READ - Recognition and Enrichment of Archival Documents), and by the Austrian Science Fund (FWF) under grant No P29892.

\section{REFERENCES}

[1] R. L. E. Jr, W. A. Christens-Barry, and K. T. Knox, "Spectral image processing and analysis of the Archimedes Palimpsest," in Proceedings of the 19th European Signal Processing Conference, EUSIPCO 2011, Barcelona, Spain, August 29 - Sept. 2, 2011, 2011, pp. 1440-1444. [Online]. Available: http://ieeexplore.ieee.org/document/7073928/

[2] Z. Khan, F. Shafait, and A. Mian, "Hyperspectral Imaging for Ink Mismatch Detection," in 2013 12th International Conference on Document Analysis and Recognition. IEEE, Aug. 2013.

[3] R. Hedjam, H. Z. Nafchi, R. F. Moghaddam, M. Kalacska, and M. Cheriet, "ICDAR 2015 contest on MultiSpectral Text Extraction (MS-TEx 2015)." IEEE, Aug. 2015, pp. 1181-1185. [Online]. Available: http://ieeexplore.ieee.org/document/7333947/

[4] B. Su, S. Lu, and C. L. Tan, "Binarization of historical document images using the local maximum and minimum," in DAS, 2010, pp. 159-166.

[5] C. Tensmeyer and T. Martinez, "Document Image Binarization with Fully Convolutional Neural Networks.” IEEE, Nov. 2017, pp. 99-104. [Online]. Available: http://ieeexplore.ieee.org/document/8269956/

[6] N. R. Howe, "A Laplacian Energy for Document Binarization," in ICDAR, 2011, pp. 6-10.

[7] I. Pratikakis, K. Zagoris, G. Barlas, and B. Gatos, "ICDAR2017 Competition on Document Image Binarization (DIBCO 2017)." IEEE, Nov. 2017, pp. 1395-1403. [Online]. Available: http://ieeexplore.iee.org/document/8270159/

[8] N. Mitianoudis and N. Papamarkos, "Document image binarization using local features and Gaussian mixture modeling," Image and Vision Computing, vol. 38, pp. 33-51, Jun. 2015. [Online]. Available: http://www.sciencedirect.com/science/article/pii/S0262885615000360

[9] M. Lettner and R. Sablatnig, "Higher order MRF for foregroundbackground separation in multi-spectral images of historical manuscripts," in Document Analysis Systems, 2010, pp. 317-324.

[10] N. Mitianoudis and N. Papamarkos, "Multi-spectral document image binarization using image fusion and background subtraction techniques," in ICIP, 2014, pp. 5172-5176.

[11] A. Hyvärinen and E. Oja, "Independent component analysis: algorithms and applications," Neural Networks, vol. 13, no. 4-5, pp. 411-430, 2000.

[12] R. F. Moghaddam and M. Cheriet, "A Multiple-Expert Binarization Framework for Multispectral Images," CoRR, vol. abs/1502.01199, 2015.

[13] L. Scharf and L. McWhorter, "Adaptive matched subspace detectors and adaptive coherence estimators," in Signals, Systems and Computers, 1996. Conference Record of the Thirtieth Asilomar Conference on, 1996, pp. 1114-1117 vol.2.

[14] C. Rother, V. Kolmogorov, and A. Blake, "'GrabCut": interactive foreground extraction using iterated graph cuts," ACM Transactions on Graphics, vol. 23, no. 3, p. 309, Aug. 2004. [Online]. Available: http://portal.acm.org/citation.cfm?doid=1015706.1015720

[15] D. Arthur and S. Vassilvitskii, "K-means++: The Advantages of Careful Seeding," in Proceedings of the Eighteenth Annual ACM-SIAM Symposium on Discrete Algorithms, ser. SODA '07. Philadelphia, PA, USA: Society for Industrial and Applied Mathematics, 2007, pp. 1027-1035. [Online]. Available: http://dl.acm.org/citation.cfm?id=1283383.1283494

[16] M. Diem, F. Hollaus, and R. Sablatnig, "MSIO: MultiSpectral Document Image BinarizatIOn," in 2016 12th IAPR Workshop on Document Analysis Systems (DAS), Apr. 2016, pp. 84-89. 
[17] F. Hollaus, M. Diem, and R. Sablatnig, "Binarization of MultiSpectral Document Images," in Computer Analysis of Images and Patterns, ser. Lecture Notes in Computer Science. Springer, Cham, Sep. 2015, pp. 109-120. [Online]. Available: https://link.springer.com/chapter/10.1007/978-3-319-23117-410 